\title{
Infodemia: excesso de quantidade em detrimento da qualidade das informações sobre a COVID-19
}

doi: 10.1590/S1679-49742020000400019

\author{
Infodemic: excess quantity to the detriment of quality of information about COVID-19 \\ Infodemia: exceso de cantidad en perjuicio de la calidad de las informaciones sobre la COVID-19
}

$\mathrm{N}$

o contexto da pandemia da COVID-19, o fenômeno denominado "infodemia" tem se destacado. 0 termo se refere a "um grande aumento no volume de informações associadas a um assunto específico, que podem se multiplicar exponencialmente em pouco tempo devido a um evento específico, como a pandemia atual. Nessa situação, surgem rumores e desinformação, além da manipulação de informações com intenção duvidosa. Na era da informação, esse fenômeno é amplificado pelas redes sociais e se alastra mais rapidamente, como um vírus" ${ }^{1,2}$

0 excesso de informações, muitas vezes conflitantes, torna difícil encontrar aquelas que são verdadeiramente úteis para orientar as pessoas, e pode dificultar a tomada de decisão por gestores e profissionais da saúde, especialmente quando não há tempo hábil para avaliar as evidências disponíveis. Ademais, o constante bombardeio de informações que alcança as pessoas por diversos meios e mídias (como televisão, rádio, computador, tablets, smartphones, jornais impressos ou eletrônicos, blogs, mídias sociais, aplicativos de conversas) acaba por sobrecarregá-las. ${ }^{3}$ Com isso, muitas vezes, as pessoas se tornam ansiosas, deprimidas, ou até mesmo exauridas e incapazes de responder às demandas que se apresentam. ${ }^{1}$

A divulgação de informações claras, consistentes e baseadas em evidências é fundamental para 0 enfrentamento à pandemia. Contudo, nas mídias sociais, qualquer pessoa pode manifestar ideias ou compartilhar notícias, muitas vezes sem embasamento científico ou fonte confiável, nem qualquer controle sobre o conteúdo. Só recentemente, proprietários e responsáveis por grandes plataformas de mídia social começaram a privilegiar fontes oficiais de informações sobre a pandemia e a bloquear conteúdo inadequado. ${ }^{4}$

De fato, a divulgação de informações falsas pode trazer consequências desastrosas para indivíduos e comunidades. No Reino Unido, foram compartilhadas massivamente teorias infundadas que atribuíam à tecnologia de telefonia móvel $5 \mathrm{G}$ a responsabilidade pela propagação do novo coronavírus. Como consequência, pessoas que acreditaram em tal falácia incendiaram quase 100 torres de telefonia móvel e agrediram funcionários das operadoras. ${ }^{5,6}$ № Irãa, onde circularam notícias falsas de que beber álcool forneceria alguma proteção contra a COVID-19 ou mataria 0 coronavírus, mais de 700 pessoas morreram após tomarem álcool de origem desconhecida e contaminado com metanol. ${ }^{7}$

No Brasil, não foram observadas situações tão extremas. Contudo, a circulação de notícias falsas é intensa. Principalmente por meio das mídias sociais - WhatsApp, Facebook e Instagram -, são disseminadas informações falsas, como a inexistência de casos de COVID-19, inclusive com imagens de leitos hospitalares desocupados, e aquelas que relatam métodos caseiros para a prevenção do contágio pelo coronavírus, tratamentos sem comprovação científica de eficácia, e teorias conspiratórias que atribuem a pandemia a uma estratégia política, com posicionamentos contrários às medidas de distanciamento social necessárias à contenção da propagação da doença. ${ }^{8}$

Tal situação é preocupante, tendo em vista que essas mídias alcançam elevada parcela da população. Ademais, a internet é importante fonte de busca de informações sobre as medidas de prevenção da COVID-19 no cenário nacional. Estudo revelou que os períodos de aumento do interesse sobre a COVID-19 ocorreram após a divulgação dos principais marcos epidemiológicos da doença no país pelos meios de comunicação. ${ }^{9}$ Além disso, os resultados sugerem possíveis lacunas de informação sobre algumas das principais formas de prevenção, o que contribui para a propagação de informações falsas. 
Desde o início da pandemia, o Ministério da Saúde (MS) tem atuado no sentido de enfrentar as notícias falsas e fornecer informações confiáveis para a população e a imprensa. ${ }^{10} 0$ site do MS (https://www.saude.gov.br/fakenews) traz uma lista de notícias falsas, o que se sabe realmente sobre 0 assunto em questão, e recomendações para a prevenção da COVID-19. Exemplos são apresentados na Figura 1. 0 MS também disponibilizou um número de WhatsApp (61 - 99289 4640) para envio de mensagens recebidas nas mídias sociais contendo informações duvidosas, cuja veracidade deverá ser apurada pelas áreas técnicas, dando-se retorno oficial, em seguida, a tais mensagens. A principal forma de minimizar a infodemia é orientar as pessoas para que verifiquem a fonte e a veracidade das informações antes de compartilhá-las ou de as utilizarem como orientação. Ou seja, deve ser priorizada a qualidade, em vez da quantidade de informações.

Em julho de 2020, a Organização Mundial da Saúde (OMS), reconhecendo a importância de responder à infodemia, promoveu a primeira conferência científica sobre o tema. ${ }^{11}$ Foram reunidos 110 especialistas, os quais concluíram que tal epidemia de desinformação requer uma resposta coordenada e multidisciplinar. Do mesmo modo que as autoridades sanitárias se apoiam na ciência da epidemiologia para a tomada de decisões na resposta à pandemia, são necessárias ferramentas e intervenções baseadas em evidências para 0 enfrentamento à infodemia, baseadas na ciência da gestão de infodemias, denominada "infodemiologia". ${ }^{12}$ Nesse contexto, foram definidos quatro pilares para a gestão das infodemias: (1) monitoramento de informações (vigilância); (2) fortalecimento da capacidade de alfabetização em saúde digital e ciência; (3) incentivo a processos de aprimoramento da qualidade das informações, como verificação de fatos e revisão por pares; e (4) tradução precisa e oportuna do conhecimento, minimizando fatores de distorção, como influências políticas ou comerciais. ${ }^{13,14}$

A OMS destaca, também, o papel dos cientistas e das revistas científicas, que têm o dever de divulgar os resultados das pesquisas de forma ampla e oportuna, e devem buscar comunicar os resultados das pesquisas de modo compreensível para a população. ${ }^{1}$ Ainda, no contexto da pandemia da COVID-19, são amplificados problemas relacionados ao desperdício em pesquisa, abrangendo perguntas de pesquisa frágeis, desenhos de pesquisa inadequados, diversas formas de ineficiência nos estudos, e relatos deficientes dos resultados, que podem contribuir para 0 agravamento da infodemia. ${ }^{15}$ Tal situação reforça a relevância do papel dos periódicos na triagem e aprimoramento da qualidade das publicações científicas.

Em alinhamento com as boas práticas na publicação científica e com as recomendações da OMS, a Epidemiologia e Serviços de Saúde: revista do Sistema Único de Saúde do Brasil (RESS) está empreendendo esforços com o intuito de publicar conteúdo de qualidade relacionado à vigilância, prevenção e controle da COVID-19 de forma oportuna e com ampla divulgação. Entre as iniciativas adotadas, destacam-se o lançamento de uma chamada para artigos sobre o tema, a aceleração do processamento de tais artigos (fast tracking) e a criação de uma página específica no sítio eletrônico da revista (https://bit.ly/30Gu7Dg). Além disso, os artigos têm sido divulgados no blog SciEL0 em Perspectiva (https://blog.scielo.org/), com a publicação de press releases.

Leila Posenato Garcia1 - (1) http://orcid.org/0000-0003-1146-2641

Elisete Duarte ${ }^{2}-\oplus$ http://orcid.org/0000-0002-0501-0190

'Instituto de Pesquisa Econômica Aplicada, Diretoria de Estudos e Políticas Sociais, Brasília, DF, Brasil

${ }^{2}$ Secretaria de Vigilância em Saúde do Ministério da Saúde, Coordenação-Geral de Desenvolvimento da Epidemiologia em Serviço, Brasília, DF, Brasil 


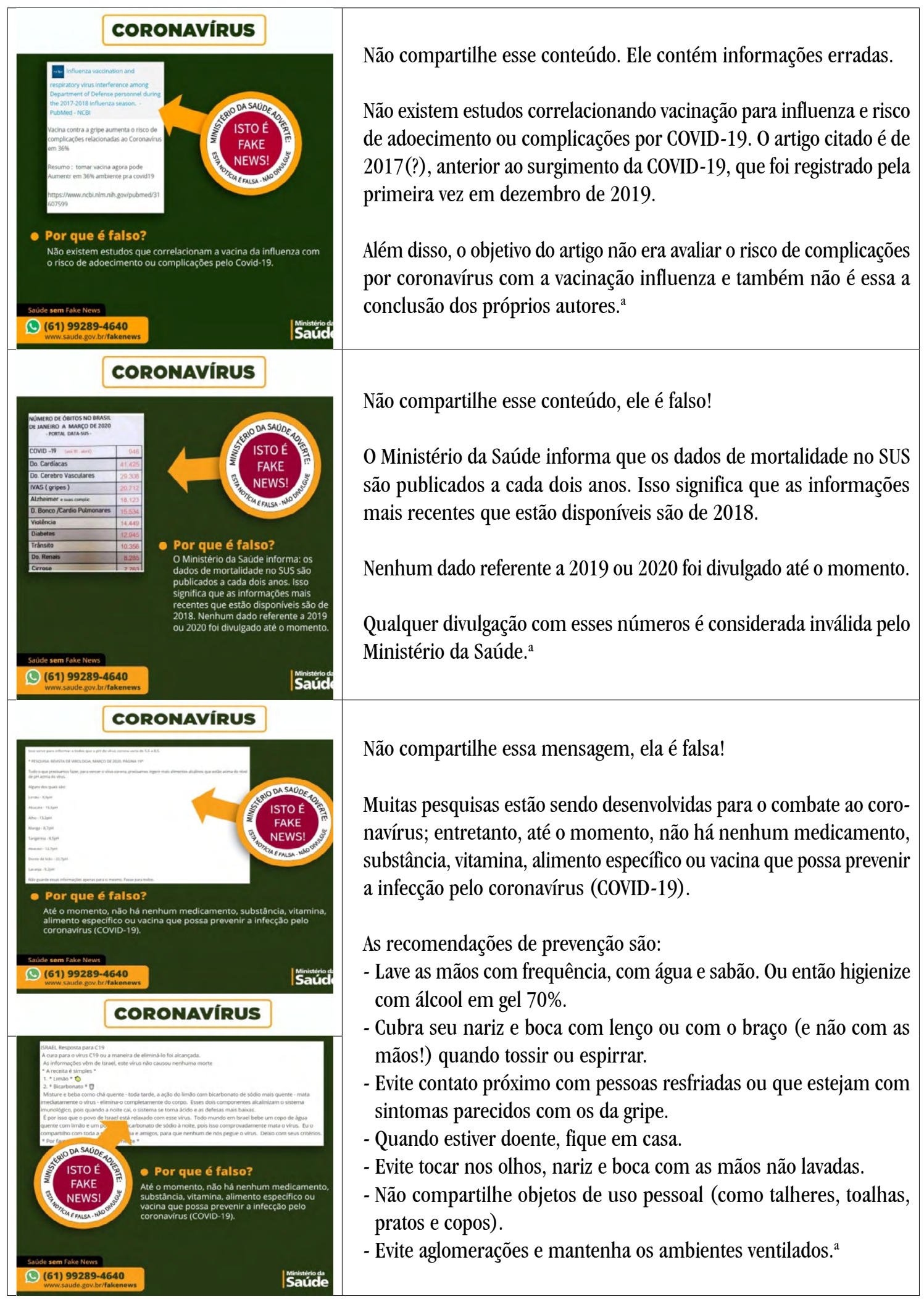

Figura 1 - Exemplos de respostas do Ministério da Saúde a notícias falsas sobre a COVID-19

Fonte:https://www.saude.gov.br/fakenews

a) Para saber mais sobre o coronavírus (COVID-19), acesse o portal oficial do Ministério da Saúde:http://saude.gov.br/saude-de-a-z/coronavirus. 


\section{Referências}

1. Organização Pan-Americana da Saúde - OPAS. Organização Mundial da Saúde - OMS. Repositório Institucional para Troca de Informações - Iris. Fichas Informativas COVID-19: entenda a infodemia e a desinformação na luta contra a COVID-19 [Internet]. Brasília: Organização Pan-Americana da Saúde; 2020 [citado 2020 ago 3]. Disponível em: https:// iris.paho.org/handle/10665.2/52054?locale-attribute $=\mathrm{pt}$

2. Zarocostas J. How to fight an infodemic. Lancet [Internet]. 2020 Feb [cited 2020 Aug 30];395(10225):676. Available from: https://doi.org/10.1016/S0140-6736(20)30461-X

3. Naeem SB, Bhatti R, Khan A. An exploration of how fake news is taking over social media and putting public health at risk. Health Inf Libr J [Internet]. 2020 Jul [cited 2020 Aug 3]. Available from: https://doi.org/10.1111/hir.12320

4. Vasconcellos-Silva PR, Castiel LD. COVID-19, as fake news e o sono da razão comunicativa gerando monstros: a narrativa dos riscos e os riscos das narrativas. Cad Saúde Pública [Internet]. 2020 jul [citado 2020 ago 3];36(7):e00101920. Disponível em: http://dx.doi.org/10.1590/0102-311X00101920

5. Jolley D, Jenny P. Pylons ablaze: Examining the role of 5G COVID-19 conspiracy beliefs and support for violence. British J Soc Psyc [Internet]. 2020 Jun [cited 2020 Aug 3];59:628-40. Available from: https://doi.org/10.1111/bjso.12394

6. Ahmed W, Vidal-Alaball J, Downing J, Lopes Segui F. COVID-19 and the 5G conspiracy theory: social network analysis of twitter data. J Med Int Research [Internet]. 2020 May [cited 2020 Aug 3];22(5). Available from: https://www.ncbi.nlm. nih.gov/research/coronavirus/publication/32352383

7. Shokoohi M, Nasiri N, Sharifi H, Baral S, Stranges S. A syndemic of COVID-19 and methanol poisoning in Iran: Time for Iran to consider alcohol use as a public health challenge? Alcohol [Internet]. 2020 Sep [cited 2020 Aug 3];87:25-7. Available from: https://doi.org/10.1016/j.alcohol.2020.05.006

8. Fundação Oswaldo Cruz - Fiocruz. Estudo identifica principais fake news relacionadas à Covid-19. Rio de Janeiro: Fiocruz; 2020 [citado 2020 ago 3]. Disponível em: https://portal.fiocruz.br/noticia/estudo-identifica-principais-fakenews-relacionadas-covid-19

9. Garcia Filho C, Vieira LJES, Silva RM. Buscas na internet sobre medidas de enfrentamento à COVID-19 no Brasil: descrição de pesquisas realizadas nos primeiros 100 dias de 2020. Epidemiol Serv Saúde [Internet]. 2020 jun [citado 2020 Jul 31];29(3):e2020191. Disponível em: https://doi.org/10.5123/s1679-49742020000300011

10. Oliveira WK, Duarte E, França GVA, Garcia LP. Como o Brasil pode deter a COVID-19. Epidemiol Serv Saúde [Internet]. 2020 abr [citado 2020 Jul 22];29(2):e2020044. Disponível em: https://doi.org/10.5123/s1679-49742020000200023

11. World Health Organization - WHO. 1st WHO Infodemiology Conference [Internet]. Geneva: World Health Organization; 2020 [cited 2020 Aug 3]. Available from: https://www.who.int/news-room/events/detail/2020/06/30/defaultcalendar/1st-who-infodemiology-conference

12. Eysenbach G. Infodemiology: the epidemiology of (mis)information. Am J Med [Internet]. 2002 Dec [cited 2020 Aug 3];113(9):763-5. Available from: hhttps://doi.org/10.1016/S0002-9343(02)01473-0

13. Eysenbach G. How to Fight an Infodemic: The Four Pillars of Infodemic Management. J Med Int Research [Internet]. 2020 Jun [cited 2020 Aug 3];22(6). Available from: https://www.jmir.org/2020/6/e21820/

14. Tangcharoensathien V, Calleja N, Nguyen T, Purnat T, D'Agostino M, Garcia-Saiso S, et at. Framework for managing the COVID-19 infodemic: methods and results of an online, crowdsourced WHO technical consultation. J Med Int Research [Internet]. 2020 Jun [cited 2020 Aug 3];22(6). Available from: https:/www.ncbi.nlm.nih.gov/research/coronavirus/ publication $/ 32558655$

15. Glasziou PP, Sanders S, Hoffmann T. Waste in covid-19 research. BMJ [Internet]. 2020 May [cited 2020 Aug 3];369:m1847. Available from: https://doi.org/10.1136/bmj.m1847 